\title{
Comparison of meconium and neonatal hair analysis for detection of gestational exposure to drugs of abuse
}

\author{
B Bar-Oz, J Klein, T Karaskov, G Koren
}

Arch Dis Child Fetal Neonatal Ed 2003;88:F98-F100

See end of article for authors' affiliations

.....................

Correspondence to: Dr Koren, Director, Motherisk Program,

Division of Clinical

Pharmacology and

Toxicology, Hospital for

Sick Children, 555

University Avenue, Toronto,

Ontario M5G 1 X8

Canada;

gkoren@sickkids.ca

Accepted 22 July 2002

\begin{abstract}
Background: Meconium and hair are two biological markers of in utero exposure to illicit drugs.
Objective: To compare the sensitivity of the two tests for different drugs.

Setting: Motherisk laboratory which tests in utero drug exposure in Toronto.

Methods: Cocaine, benzoylecgonine, opiates, cannabis, benzodiazepines, methadone, and barbiturates were measured in pairs of hair and meconium samples from the same neonates.

Results: Meconium was marginally more sensitive than neonatal hair for detection of cocaine and cannabis, possibly because it may detect second trimester exposure whereas hair grows only during the third trimester of pregnancy. There was a significant correlation between hair and meconium concentrations of cocaine, cannabis, and opiates.

Conclusion: In cases of clinical suspicion and a negative neonatal urine test, both meconium and hair are effective biological markers of in utero illicit drug exposure. Meconium may be more sensitive, but neonatal hair is available for three months whereas meconium is available for only one or two days. In contrast, the use of meconium, being a discarded material, is more acceptable to some parents than hair testing, which entails cutting scalp hair from the newborn.
\end{abstract}

D uring the past two decades, illicit drug use has reached epidemic proportions in North America. ${ }^{1}$ In the United States, $10-45 \%$ of the women cared for at urban teaching hospitals use cocaine during pregnancy. ${ }^{2}$ As women of reproductive age constitute a large segment of the drug using population, the effects of their drug use on the fetus has been studied extensively. Prenatal cocaine use has been associated with placental abruption and premature labour, ${ }^{3-5}$ as well as with increased rates of low birth weight, microcephaly, congenital anomalies, and necrotising enterocolitis. ${ }^{67}$ However, because of multiple other reproductive risk factors in women using illicit drugs, it is possible that many of the adverse effects attributed to drugs are caused by other factors. ${ }^{5}$ Similarly, it is not clear whether cocaine per se, or other risk factors, leads to adverse neurobehavioural effects. ${ }^{8}$ Of importance, maternal addiction itself is a determinant of serious postnatal risk for the infant. Newborns exposed to opioids, barbiturates, benzodiazepines, or alcohol in utero may experience withdrawal symptoms, often requiring treatment. $^{910}$

Estimated rates of infants exposed prenatally to cocaine range between $2.6 \%$ and $11 \%$ of all live births. ${ }^{11}$ A prevalence study of cocaine use during pregnancy conducted by our group in 1990-1991 in three Metropolitan Toronto hospital nurseries (one inner city, two suburban) found 37 out of 600 (6.25\%) infants tested positive for cocaine. ${ }^{12}$ In the Metropolitan Toronto area, there has been a steady increase in the number of newborns affected by maternal drug use. ${ }^{13}$

It has been shown that maternal reporting of drug use is far from accurate. ${ }^{12}{ }^{14}$ Fearing legal consequences and embarrassment from admitting illicit substance use, most users tend to deny or to under-report drug consumption.

A major problem in studying the adverse effects of illicit drugs is the lack of standardised techniques to ascertain fetal exposure. The validity of blood and urine tests depends on the elimination half life of the compound in question. In the case of cocaine, which has a short elimination half life of less than one hour, the drug and its metabolites are not likely to be detected for more than a few days in either blood or urine. ${ }^{14}$ Other drugs, such as cannabis and opioids, have longer elimi- nation half lives, but even these drugs can be detected for only a maximum of three to four weeks after use. ${ }^{15}$

These facts have highlighted an urgent need for a biological marker which will still be sensitive weeks after the end of exposure and which may yield a cumulative reflection of long term exposure to illicit drugs.

In 1989, we first reported the use of hair analysis as a biological marker for gestational cocaine exposure in seven neonates whose mothers were known cocaine users. ${ }^{16}$ This test is widely used at present. Meconium testing has proved to be another very effective tool for verifying gestational drug exposure. ${ }^{14}$ No formal comparison of the sensitivities of meconium and hair analysis for different drugs of abuse has as yet been conducted.

The aim of this study was to estimate the sensitivity and correlation between neonatal hair and meconium testing in 185 infants suspected of being exposed in utero to one or more illicit substances, namely cocaine, opiates, and cannabis.

\section{SUBJECTS AND METHODS}

Between 1999 and September 2001 the Motherisk laboratory at the Hospital for Sick Children in Toronto, Canada received thousands of neonatal hair and meconium samples for analysis. Among them were 185 pairs of hair and meconium samples collected from the same babies in various hospitals in Ontario. Based on clinical suspicion of maternal drug abuse, the testing of hair and/or meconium was requested by either a doctor or the Children's Aid Societies. Most of the requests were for analysis for cocaine, opiates, and cannabinoids. We report on measurements of cocaine, its metabolite benzoylecgonine, heroin, morphine, cannabis, methadone, benzodiazepines, and barbiturates.

\section{Hair testing}

Hair was analysed by well established methods. ${ }^{17}$ It was not washed before testing unless external contamination was suspected. If washing of the hair sample was included in the procedure, a previously described method was followed. ${ }^{18}$

Briefly, 2-5 mg finely cut hair, unwashed or previously washed, was sonicated in $1 \mathrm{ml}$ methanol/5 $\mathrm{M} \mathrm{HCl}(20: 1, \mathrm{v} / \mathrm{v})$ 
Table 1 Proportion of positive/negative tests for cocaine, benzoylecgonine, opiates, and cannabinoids in neonatal hair and meconium

\begin{tabular}{lllll}
\hline & Cocaine & Benzoylecgonine & Opiates & Cannabinoids \\
\hline + meconium, + hair & 41 & 35 & 19 & 33 \\
+ meconium, - hair & 10 & 15 & 4 & 21 \\
- meconium, + hair & 2 & 3 & 4 & 1 \\
Total positive sample & 53 & 53 & 27 & 55 \\
- meconium, - hair & 120 & 119 & 109 & 86 \\
Total samples tested & 173 & 172 & 136 & 141 \\
\hline
\end{tabular}

for 30 minutes and incubated overnight at $45^{\circ} \mathrm{C}$. On the next day, the methanol was pipetted off, and the hair rinsed briefly with an additional $1 \mathrm{ml}$ methanol. After evaporation of the methanol at $40^{\circ} \mathrm{C}$ under a stream of nitrogen, $200 \mu \mathrm{l}$ phosphate buffered saline at $\mathrm{pH}$ 7.0-7.4 was added, and the individual drugs were analysed by enzyme linked immunosorbent assay using kits manufactured by Immunalysis (San Diego, California, USA).

For quantification, standards were prepared in blank hair extract to control for matrix effect. Different blank hair extracts were used to match the age and hair colour of the subject. The limit of detection for each drug was $0.2 \mathrm{ng} / \mathrm{mg}$ hair when $2 \mathrm{mg}$ hair was used. Positive results were confirmed using gas chromatography/mass spectrometry with the mass selective detector operating in selective ion monitoring mode.

\section{Meconium testing}

For meconium testing, approximately $0.2 \mathrm{~g}$ wet meconium was extracted with methanol. After centrifugation, the supernatant was diluted 1:5 with phosphate buffered saline, and an aliquot was analysed for cocaine, benzoylecgonine, opiates, and/or cannabinoids. Standards were prepared in blank meconium extract similarly to the hair samples. Similar immunoassays were used to those for the hair analysis described above. Here too, positive results were confirmed by gas chromatography/mass spectrometry. The limit of detection for each drug was $50 \mathrm{ng} / \mathrm{g}$ meconium when $0.2 \mathrm{~g}$ meconium was used for testing. The coefficient of variation of these tests in our laboratory is less than $5 \%$.

\section{Statistical analysis}

Assuming a false positive rate of zero for all the drugs analysed-that is, a specificity of $100 \%$-the estimated sensitivity of the test was calculated for each drug, with the rate of positive hair plus meconium in neonates estimated to represent $100 \%$ of positive cases.

Linear regression and Spearman rank order correlation were used whenever appropriate.

\section{RESULTS}

Of 185 pairs of hair and meconium samples assayed, 75 were negative for all the drugs analysed. Table 1 shows the distributions of cocaine, benzoylecgonine, opiates, and cannabis in the positive hair and meconium samples. A total of 173 pairs were tested for cocaine, 172 pairs for benzoylecgonine, 136 pairs for opiates, and 141 pairs for cannabis. Additional tests were performed for benzodiazepines (two pairs), methadone (two pairs), and barbiturates (one pair).

\section{Cocaine testing}

The total number of positive samples for cocaine (hair, meconium or both) was 53, of which 51 were positive in meconium whereas 43 were positive in hair. The calculated sensitivity for cocaine testing was $96 \%$ in meconium and $84 \%$ for hair (table 2).
Table 2 Sensitivity of neonatal hair and meconium for drugs of abuse

\begin{tabular}{lll}
\hline & $\begin{array}{l}\text { Meconium testing } \\
\text { sensitivity }\end{array}$ & $\begin{array}{l}\text { Hair testing } \\
\text { sensitivity }\end{array}$ \\
\hline Cocaine & $96 \%$ & $84 \%$ \\
Benzoylecgonine & $95 \%$ & $78 \%$ \\
Opiates & $87 \%$ & $87 \%$ \\
Cannabis & $98 \%$ & $71 \%$ \\
\hline
\end{tabular}

Table 3 Results of neonatal hair and meconium analysis for benzodiazepines, methadone, and barbiturates

\begin{tabular}{llll}
\hline & $\begin{array}{l}\text { Total } \\
\text { number }\end{array}$ & $\begin{array}{l}\text { Positive in } \\
\text { meconium }\end{array}$ & $\begin{array}{l}\text { Positive in } \\
\text { hair }\end{array}$ \\
\hline Benzodiazepines & 2 & 2 & 2 \\
Methadone & 2 & 2 & 2 \\
Barbiturates & 1 & 1 & 1 \\
\hline
\end{tabular}

The total number of positive samples for benzoylecgonine was 53, of which 50 were positive in meconium (sensitivity of $95 \%$ ), and 38 were positive in hair ( sensitivity of $78 \%$ ).

There was a significant correlation between hair and meconium levels of cocaine $\left(r_{0}=0.83, \mathrm{p}<0.001\right)$ and benzoylecgonine $\left(r_{0}=0.56, \mathrm{p}<0.001\right)$.

\section{Opiate testing}

The total number of positive samples was 27, of which 23 were positive in both or one of the matrices studied (sensitivity $87 \%$ ). There was a highly significant correlation between hair and meconium levels $\left(r_{0}=0.69, \mathrm{p}<0.0001\right)$.

\section{Cannabis testing}

The total number of positive samples was 55, of which 54 were positive in meconium and 34 were positive in hair. The calculated sensitivity for cannabis in meconium was $98 \%$, and for hair it was $71 \%$. There was a highly significant correlation between hair and meconium measures $\left(r_{\mathrm{o}}=0.73\right.$, $\mathrm{p}<0.0001)$.

\section{Miscellaneous testing}

Two hair-meconium pairs were positive for benzodiazepines in both matrices, methadone was positive in hair and in meconium in two pairs tested, and one pair was positive for barbiturates (table 3 ).

\section{DISCUSSION}

As illicit drug use reaches epidemic proportions, protecting the wellbeing of the fetus and offspring of drug users is a serious challenge for health professionals and social services. A positive meconium test can reflect maternal use of illicit drugs 
from the second trimester of pregnancy onwards. Only meconium collected during the first 1 or 2 days of life or for the first three stools can be used to document in utero drug exposure. In contrast, neonatal hair, which grows during the third trimester, may reflect exposure of drugs during the last trimester of pregnancy, and can stay positive for up to three months after birth.

This study shows the correlation between meconium-hair pairs for cocaine, benzoylecgonine, opiates, and cannabis. For cocaine, benzoylecgonine, and cannabis, meconium testing seems to be more sensitive ( $95 \%$ and above) than hair testing. This may be partly explained by the earlier formation of meconium compared with hair (roughly the second trimester compared with the third trimester). The limitation of using meconium for routine testing is the narrow time frame for obtaining the sample. In all the cases in which meconium tested negative whereas the hair tested positive, although the documents specified the sample to be meconium, in fact it was a mixture of meconium and stool, confirming that, for the test to be accurate, meconium has to collected not later than one or two days after birth.

Chiriboga et al ${ }^{19-21}$ have shown a concentration-response effect of cocaine, as measured in maternal hair, on newborn head circumference and abnormalities in muscle tone, movement, and posture. Our study is the first to document significant correlation between meconium and hair levels of different illicit drugs, which enables the use of either meconium or neonatal hair for assessment of the magnitude of exposure and therefore the expected neurological impairment to the exposed newborn. It is possible that a combination of negative hair test and positive meconium test reflects second trimester exposure to drugs, with no third trimester exposure.

Benzodiazepines are thought to be human teratogens, possibly causing oral clefts. ${ }^{22}$ Although meconium forms only in the second trimester, it is important to document the pattern of benzodiazepine use in cases of oral cleft. Both meconium and hair can be used for this purpose.

Maternal abuse of barbiturates can cause abstinence syndrome in the newborn infant. Sampling either meconium or hair, or both, can help to establish the diagnosis when the maternal history is not accurate or available.

Both meconium and hair analysis have advantages and disadvantages. Meconium may be more sensitive. However, it is available for only two days after birth, whereas hair may be available for up to three months. Because meconium production begins in weeks 14-16, meconium testing may detect second trimester exposure to drugs, whereas the hair present at birth only develops in the third trimester. Although this may increase the sensitivity of the meconium test, third trimester exposure, evidenced by hair testing, reflects drug abuse long after pregnancy was detected and hence is diagnostic of maternal addiction, which has important implications for neonatal care. Some parents resist hair cutting, whereas meconium is a discarded material. Equally as important, some babies are born with very little hair or no hair at all. Another problem with hair analysis is that hair levels of drugs are affected by the amount of melanin in the shaft. ${ }^{23}$ The dose-response characteristics of deposition of drugs in hair and meconium have been documented..$^{19} 2024$

To improve the yield of both matrices involved, we propose to initiate clinical investigations with urine testing in suspected cases. If the urine test is negative, meconium or hair testing will be used depending on postnatal age. In cases with a high index of suspicion, both matrices should be used, rendering higher sensitivity. Because of the strong correlation between their measured levels, both matrices can be used to estimate the extent and timing of fetal exposure and the resulting neurological impairment.

\section{ACKNOWLEDGEMENTS}

This work was supported by a grant from The Canadian Institute for Health Research (CIHR), and the Research Leadership in Better Pharmacotherapy During Pregnancy and Lactation. BB-O was a recipient of a Fellowship award by the Research Training Center, The Hospital for Sick Children. GK is a Senior Scientist of CIHR.

\section{Authors' affiliations}

B Bar-Oz, J Klein, T Karaskov, G Koren, The Motherisk Program, Division of Clinical Pharmacology and Toxicology, Hospital for Sick Children and University of Toronto, Toronto, Canada

B Bar-Oz, Department of Neonatology, Hadassah Medical Center and The Hebrew University, Jerusalem, Israel

\section{REFERENCES}

1 Jekel JF, Allen DF, Podlewski $\mathrm{H}$, et al. Epidemic freebase cocaine abuse. Lancet 1980;1:459-62.

2 Volpe JJ. Effect of cocaine use on the fetus. N Engl J Med 1992;327:399-407.

3 Gillogley KM, Evans AT, Hansen RL, et al. The perinatal impact of cocaine, amphetamine and opiate use detected by universal intrapartum screening Am J Obstet Gynecol 1990;163:1535-42.

4 Chasnoff IJ, Griffith DR. Cocaine: clinical studies of pregnancy and the newborn. Ann NY Acad Sci 1989:562:260-6.

5 Addis A, Moretti ME, Syed FA, et al. Fetal effects of cocaine: an updated meta-analysis. Reprod Toxicol 2001;15:341-69.

6 Chasnoff IJ, Bussey ME, Savich R, et al. Perinatal cerebral infarction and maternal cocaine use. J Pediatr 1986;108:456-9.

7 Lopez SL, Taeusch HW, Findlay RD, et al. Time of onset of necrotizing enterocolitis in newborn infants with known prenatal cocaine exposure. Clin Pediatr (Phila) 1995;34:424-9.

8 Frank DA, Augustyn M, Knight WG, et al. Growth, development, and behaviour in early childhood following prenatal cocaine exposure; a systematic review. JAMA 2001;285:1613-25.

9 Franck L, Vilardi J. Assessment and management of opioid withdrawal in ill neonates. Neonatal Netw 1995;14:39-48.

10 Dixon SD. Effects of transplacental exposure to cocaine and methamphetamine on the neonate. West J Med 1991;150:436-42.

11 Birchfield M, Scully J, Handler A. Perinatal screening for illicit drugs: policies in hospitals in a large metropolitan area. J Perinatol 1995; 15:208-14.

12 Forman R, Klein J, Meta D, et al. Maternal and neonatal characteristics following exposure to cocaine in Toronto. Reprod Toxicol 1993; 7:619-22

13 Metro Toronto Research Group on Drug Use in Metropolitan Toronto. Toronto: The Metro Toronto Department of Health, 1995:51.

14 Ostrea EM, Knapp DK, Tannenbaum L, et al. Estimates of illicit drug use during pregnancy by maternal interview: hair analysis and meconium analysis. J Pediatr 2001;138:344-8.

15 Cirimele V, Kintz P, Mangin P. Testing human hair for cannabis. Forensic Sci Int 1995;70: 175-82.

16 Graham K, Koren G, Klein J, et al. Determination of gestational cocaine exposure by hair analysis. JAMA 1989;262:3328-30

17 Klein J, Karaskov T, Koren G. Clinical applications of hair testing for drugs of abuse: the Canadian experience. Forensic Sci Int 2000; 107:281-8

18 Koren G, Klein J, Forman R, et al. Hair analysis of cocaine: differentiation between systemic exposure and external contamination. J Clin Pharmacol 1992:32:671-5.

19 Chiriboga CA, Bateman DA, Brust JC, et al. Neurologic findings in neonates with intrauterine cocaine exposure. Pediatr Neurol 1993;9:115-19.

20 Chiriboga CA, Brust JCM, Bateman DA, et al. Dose-response effect of fetal cocaine exposure on newborn neurologic function. Pediatrics 1999; 103:79-85

21 Bateman DA, Chiriboga CA. Dose-response effect of cocaine on newborn head circumference. Pediatrics 2000;106:e33.

22 Dolovich LR, Addis A, Regis Vaillancourt JM, et al. Benzodiazepine use in pregnancy and major malformations or oral clefts: meta-analysis of cohort and case-control studies. BM 1998:317:839-43.

23 Ursiti F, Klein J, Sellers E, et al. Use of hair analysis for confirmation of self-reported cocaine use in users with negative urine tests. J Toxicol Clin Toxicol 2001;539:361-6.

24 Delaney-Black V, Covington C, Ostrea E, et al. Prenatal cocaine and neonatal outome: evaluation of dose-response relaitionship. Pediatrics 1996;98:735-40. 